\title{
Prefrontal cortex gyrification index in twins: an MRI study
}

\author{
Alkomiet Hasan • Andrew M. McIntosh • Uta-Aglaia Droese • Thomas Schneider-Axmann • \\ Stephen M. Lawrie - Thomas William Moorhead • Ralf Tepest - Wolfgang Maier • \\ Peter Falkai $\cdot$ Thomas Wobrock
}

Received: 3 December 2010/ Accepted: 3 February 2011/Published online: 19 February 2011

(C) The Author(s) 2011. This article is published with open access at Springerlink.com

\begin{abstract}
Cortical development and folding seems to be under environmental as well as genetic control. The aim of our study was to estimate the genetic influence on gyrification and cortical volumes, comparing prefrontal gyrification index (GI) in monozygotic (MZ) and dizygotic (DZ) twin pairs, and unrelated pairs. Twenty-four subjects $(6$ pairs of MZ and 6 pairs of DZ twins) were included in this study. Prefrontal cortical folding (gyrification) was measured by an automated and manual version of the gyrification index (A-GI, M-GI) according to previously published protocols. MR-imaging was performed and 3 representative slices were selected from coronar MRimaging scans. The volumes of the total brain, temporal lobes, prefrontal lobes, and cerebellum were analyzed, too. To evaluate similarity in GI, absolute differences in GI, and brain volumes as well as intraclass correlations of twin pairs were compared with regard to twin status. Finally, a control group of unrelated pairs was assembled from the first two study groups and analyzed. Compared to unrelated
\end{abstract}

A. Hasan ( $\square)$. U.-A. Droese · T. Schneider-Axmann .

P. Falkai - T. Wobrock

Department of Psychiatry and Psychotherapy,

Georg-August-University, Von-Siebold-Str. 5,

37075 Göttingen, Germany

e-mail: ahasan@gwdg.de

A. M. McIntosh - S. M. Lawrie - T. W. Moorhead Department of Psychiatry, University of Edinburgh, Edinburgh, United Kingdom

R. Tepest

Department of Psychiatry and Psychotherapy,

University of Cologne, Cologne, German

W. Maier

Department of Psychiatry and Psychotherapy,

University of Bonn, Bonn, Germany pairs, twin pairs exhibited more similarity concerning different brain volumes and a trend to more similarity concerning A-GI. MZ twins did not present more similarity concerning GI (automatically and manually measured) and volume measurements compared to DZ twins. Different factors, like intrauterine factors, postnatal development conditions, and especially environmental factors might account for the differences between related and unrelated pairs. The nonexistence of a pronounced similarity in MZ twins compared to DZ twins concerning prefrontal GI raises questions about the extent of genetic influence on GI.

Keywords Twins - Cortical folding - Genetics . Gyrification · Prefrontal lobes

\section{Introduction}

Cortical folding serves as a marker of late ontogenetic brain development in mammalians. The high level of gyrification in humans is associated with an increase in overall cerebral size and reflects an enlargement of cerebral surface area. The gyrification pattern is largely complete by birth and displays smooth, continuous development. Gyri and sulci appear in a specific order: the least variable "primary" gyri are first to appear, followed by the moderately variable "secondary" gyri then finally, the most variable "tertiary" gyri develop [1]. In humans, the sulcogyral development continues until early adulthood. Although maximal GI values are reached in infancy, a process of "compensation" allows for continued growth to be accommodated by compensatory tertiary folding which maintains GI levels [1]. The formation of sulcal and gyral patterns appears to be a genetically programed and environmentally influenced event [2]. Different studies point to 
a fractional genetic determined composition of sulcal and gyral structures. One study group explored brain volumes and external sulcal patterns in monozygotic (MZ) and dizygotic (DZ) twins and found contribution of genes in cortical gyral patterns [3]. Their results indicate that gyral patterns are significantly more alike within $\mathrm{MZ}$ twin pairs than within DZ twin pairs, whereas the heritability of brain volume is more distinctive than the heritability of sulcal variation [3]. Lohmann et al. [4] reported that human sulcal patterns are significantly more alike in $\mathrm{MZ}$ twins than in unrelated and matched pairlings and that the shape of deep (ontogenetically early) sulci of the human brain is more strongly predetermined than the superficial sulci. These findings lead to the conclusion that the cerebral gyrification and the shape of the human brain might be a largely genetically determined process.

To investigate the mainly unknown mechanisms of gyral and sulcal ontogenesis, twin studies have been of particular significance [4] and several studies have reported highly significant correlations between healthy MZ twins for midline structures, total brain volume, total brain surface area and various cerebral substructures [5]. Compared to this midline structures and brain volumes, the patterns of gyral and sulcal development seemed to have more variability in twins [3, 4, 6], indicating that cortical surface development is under greater nongenetic influence than other brain measures [7]. However, the processes underlying the development of cortical convolution in healthy and mental ill persons and their relationship to cortical regionalization remain essentially unknown [8] and only few studies did survey similarity of twin brain. Changes in cortical surface and especially in prefrontal gyrification have been recently observed in different, highly heritable psychiatric diseases, like schizophrenia [9] or obsessivecompulsive disorder [10]. These findings have been interpreted as a hint toward an abnormal neurodevelopment of brain structures in these psychiatric diseases, and gyrification changes are discussed to be an endophenotypic marker, whereas strong evidence is lacking.

The aim of our study was to determine the genetic influence on prefrontal cortical folding using the Gyrification index (GI) in order to compare prefrontal gyrification between MZ and DZ twins. The GI is a ratio of the inner and the outer contour of the brain [11] and it increases proportionally to the number of gyri [12].

Two different procedures of measuring GI are available. In the present study, we used an automated [13] and a manual method [14] to determine the gyrification index for the comparison of MZ and DZ twin pairs and unrelated pairs. This procedure was chosen to gaze specifically at prefrontal gyrification by using the M-GI method and to minimize haziness of the A-GI method, which can be lead back to the fact that A-GI is applied to every coronal slice.
Different studies have pointed out the similarity in brain morphology in MZ and DZ twins, but to our knowledge our study is the first exploring the effects of heritability on cortical surface in healthy MZ and DZ twins using two different, well-validated methods, for calculating GI. With regard to the above cited literature, we set up the hypothesis that MZ twins would present more similar GI and brain volumes than DZ twins. Compared to unrelated pairs, we assumed that twin pairs would present more similarity in the mentioned target regions.

\section{Materials and methods}

Subjects

In total 24 subjects were included in this study. Six male MZ twin pairs (mean age 27.2 years, range 19-36 years) and six male DZ (mean age 26.7 years, range 24-29 years) twin pairs participated in the study and none of the subjects had a history of neurological or psychiatric disorders. To evaluate unrelated pairs within the sample, we composed age-matched unrelated pairs from our two study groups. After a complete description of the study, written informed consent was obtained from each subject. The local ethics committees approved the protocol, which was in accordance to the Declaration of Helsinki.

\section{MRI acquisitation}

MRI scanning was performed on a 1.5 Tesla Philips S15ACS using a T1-weighted, FLASH-3-sequence $(17 \mathrm{~ms}$ repetition time, $5 \mathrm{~ms}$ echo time, $35^{\circ}$ angle) with 53 consecutive slices of $3 \mathrm{~mm}$ thickness and a pixel size of $0,449 \times 0,449 \mathrm{~mm}$. Prior to analysis, scans were realigned so that the coronal plane was orthogonal to the AC-PC line.

Image processing and analysis used the software packages Analyze (Mayo Foundation, 1999), SPM as well as own IDL applications. Details on image preprocessing operations were described in previous work [13]. Briefly summarized, the SPM package (http://www.fil.ion.ucl.ac. $\mathrm{uk} / \mathrm{spm} /$ ) [15] was used to perform preprocessing functions on images used in this study. The $T_{1}$ weighted scans were segmented in native space using the SPM segment function, after which the SPM brain extraction function recovered a tissue mask for each scan. These masks were combined with the native $\mathrm{T} 1 \mathrm{~s}$ to give each brain a $\mathrm{T} 1$ tissue image with nonbrain tissue and CSF removed. The SPM coregistration function provided a mapping of the extracted brains into the Montreal Neurological Institute (MNI) space to obtain AC-PC registration while maintaining the native space volumes. This mapping into the MNI space without size or shape adjustment was applied to 
the original $\mathrm{T} 1$, the brain tissue mask, and the three native space segments. These AC-PC registered images were then resliced to $1 \times 1 \times 1 \mathrm{~cm}$ voxel size, and they formed the base data structure upon which Automated-Gyrification Index (A-GI) was implemented. Volumes of total gray matter (GM), total white matter (WM), and cerebrospinal fluid (CSF) were obtained as per SPM voxel-based morphometry (VBM) protocols using a MATLAB algorithm [15].

Automated-gyrification index (A-GI) processing

A full description of the A-GI process was given from our and other study groups elsewhere [10, 13, 16]. Briefly, the process can be divided into two procedures, the prefrontal lobe detection and the A-GI extraction. In the first procedure, an automated parcellation procedure is used to locate the left and right prefrontal lobes. In the second automated procedure, the inner and outer contours are traced. In A-GI, the inner trace is a composite of the exposed surface and closed sulci tracings. The closed sulci tracings are marked in yellow. The exposed surface is marked by a blue trace on the right side and a green trace on the left side. Where the left and right exposed surfaces overlap in the interhemisphere fissure a red trace is used. The length of the inner trace in the right side is given by a sum of the traces in red, blue, and two times the yellow (right hemisphere). Similarly, the left side inner is given by a sum of red, green, and twice the yellow (left hemisphere). This outer contour overlaps the inner contour exposed surface traces, except where are open sulci occur near the cortical surface. The outer contour is calculated for the left and right hemispheres by following this red trace. Once this has been done, the GI values are calculated for each slice and exported to a tab-delimited text file format. Measurements were taken on every $1 \mathrm{~mm}$ prefrontal slice, approximating 40 slices per brain. The GI values for each slice within the region were summed and then divided by the number of slices, producing a mean GI value for each individual (see Fig. 1).
Manual gyrification index (M-GI) processing

As described previously $[9,14]$, scans were realigned in the sagittal view resulting that the coronal plane was orthogonal to the AC-PC line. In coronal and axial view, the brains were aligned with respect to the midsagittal line. The measurements were performed with the software MRIcro (Chris Rorden, University of South Carolina, http://www.cabiatl. com/mricro/). In accordance with our and others previous work [9, 14, 17], slices for M-GI measurements of the prefrontal lobe were selected. In sagittal view, the first slice anterior to the genu of the corpus callosum vertical to the AC-PC-line was chosen as index slice for each subject. Two additional prefrontal lobe slices, 10 and $20 \mathrm{~mm}$ anterior to the index slice, were selected as well.

In coronal view, the outer and inner contour of prefrontal lobe was outlined manually in the identified slices including local maxima and minima of gyral and sulcal crests. The number of voxels (size $1 \mathrm{~mm}^{3}$ ) marking the contours was counted and the M-GI was calculated as the ratio of the inner and the outer contour (number of voxels) [11].

Ten slices were selected randomly and M-GI measured twice presenting high intra rater reliability (right M-GI: $r=0.72$, ICC $=0.74$ and left M-GI: $r=0.98$, ICC $=$ 0.995).

\section{Statistics}

All statistical testing was conducted with SPSS for Windows 17 (SPSS Inc., Chicago, Illinois). Level of significance was set at $\alpha=0.05$. Dependent variables were right and left GI in each subject for both methods (A-GI and $\mathrm{M}-\mathrm{GI}$ ), whole brain volume and absolute and relative prefrontal lobe, temporal lobe and cerebellum volume.

The sample consisted of 12 twin pairs (6 MZ, $6 \mathrm{DZ})$. As a permutation from these pairs an age-matched sample consisting of 12 unrelated pairs was assembled. First, for each independent variable Pearsons product moment correlations and intraclass correlation coefficients (ICC) were calculated for the unrelated pairs, testing the hypothesis that ICC was
Fig. 1 GI-Automated tracking, modified according to [10, 13] the following: a Total inner contour trace on a coronal slice of a subject. Colors blue exposed inner contour right, green exposed inner contour left, yellow buried inners. $\mathbf{b}$ Total outer contour trace on a coronal slice of a subject. Inner contours overlaid with smoothed outer and interhemisphere fissure trace in red

larger than zero in this age-matched sample. Second, ICC was calculated for the group of all twin pairs. It was tested, if the ICC of the related twin pairs was larger than the observed ICC of the unrelated pairs (in case of being positive). In the case of a negative ICC in unrelated pairs, it was tested if the ICC of the twin pairs was larger than zero.

A type I error probability adjustment for the number of statistical tests was applied according to an improved Bonferroni method by Hommel [18] based on Simes' test.

In additional analyses, all dependent variables ICC were calculated separately in MZ and DZ twin pairs. The extent of ICC in MZ was compared to the extent of ICC in DZ twins.

\section{Results}

Age and gender characteristics

All subjects were male. Mean age of the subjects was 27.0 years. In unrelated pairs, there were no significant differences between the ages of the first and the second subject of the pairs $(F=0.1, d f=1,11, P=0.80)$ and the correlation was high $(\mathrm{ICC}=0.96, d f=11,11$, $P<0.0005)$.

\section{Comparison of A-GI and M-GI}

The mean value of A-GI (right A-GI $=2.265$, $\mathrm{SD}=0.132$, left $\mathrm{A}-\mathrm{GI}=2.137, \quad \mathrm{SD}=0.133)$ differed from the mean value of $\mathrm{M}-\mathrm{GI}$ (right $\mathrm{M}-\mathrm{GI}=1.564$, $\mathrm{SD}=0.146$, left $\mathrm{M}-\mathrm{GI}=1.527, \quad \mathrm{SD}=0.129)$ in the whole sample. Every manually measured GI was significant smaller than an automatically measured GI. See Fig. 2 for distributions of GI measurements in the different groups.

Comparison of prefrontal GI between the MZ and DZ twins

Comparing the two groups of twin pairs A-GI did not show more similarity in $\mathrm{MZ}$ twins (right A-GI: $\mathrm{ICC}=0.46$; left
Fig. 2 Distributions of the gyrification index between twins. a Automated-GI and b manual-GI
A

Distribution of A-GI in monozygote and dizygote twins
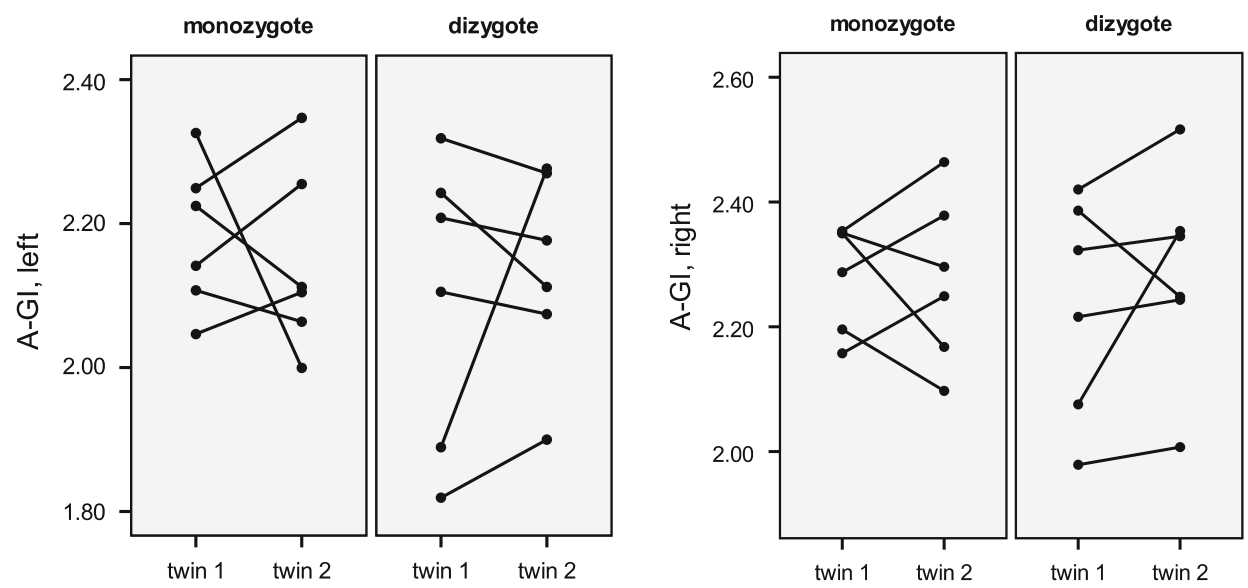

Distribution of M-GI in monozygote and dizygote twins

B

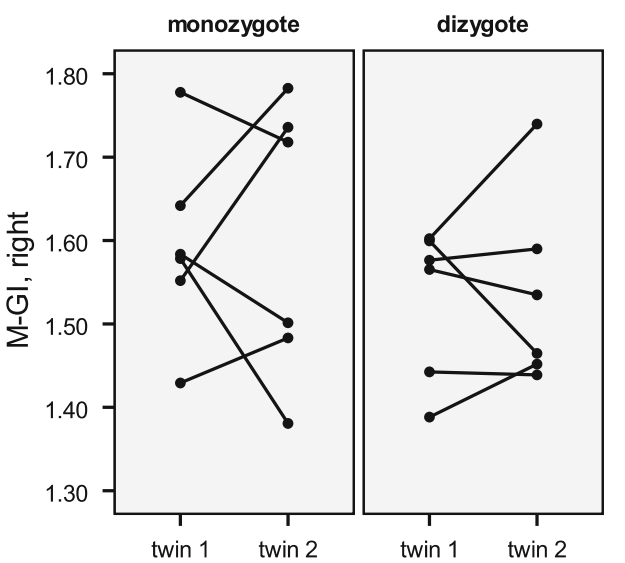


A-GI: ICC $=-0.03$ ) compared to DZ twins (right A-GI: ICC $=0.70$; left A-GI: ICC $=0.47$ ). Regarding the A-GI, MZ twins did not exhibit more similarity (right A-GI: ICC $=0.52$; left A-GI: ICC $=0.69$ ) compared to DZ twins (right A-GI: ICC $=0.65$; left A-GI: ICC $=0.61$ ).

Comparison of prefrontal GI between related and unrelated pairs

In unrelated pairs, ICCs were not significantly larger than zero. There were indications that similarity in A-GI and M-GI was larger in the twin pairs than in unrelated pairs, but only without adjustment for multiple testing. For details see Table 1.

Comparison of absolute and relative brain volumes between related and unrelated pairs

In unrelated pairs, ICC was not significantly larger than zero. ICCs were significantly larger in twin pairs compared to unrelated pairs concerning whole brain volume, absolute cerebellum volume, relative temporal lobe volume left, absolute prefrontal lobe volume right, relative prefrontal lobe volume left.

For all other brain volumes, ICC was either larger in twin pairs than in unrelated pairs, but did not reach significant without correction for multiple testing or ICC did not differ between groups. For details see Table 1 .
Absolute and relative brain volumes comparing MZ and DZ twins

Comparing absolute total brain volume and absolute as well as relative volumes of right and left temporal lobe, right and left prefrontal lobe, and cerebellum did not reveal any significant differences between MZ twins and DZ twins.

\section{Discussion}

In the present study, we tried to investigate the impact of heritability on prefrontal gyrification index in a twin sample. Therefore, we compared MZ with DZ twins and related twin pairs with unrelated pairs. Our main finding was that MZ twins did not reveal more similarity concerning GI (automatically and manually measured) than DZ twins. Another finding is the nonexistent volume difference of different brain structures in our study group, which is in contrast to other studies referring to highly significant correlations between MZ twins for different brain structures and the brain volume. However, compared to unrelated pairs, related twin pairs showed more similarity concerning A-GI (without statistical correction) and the volumes of different brain areas, which is a well-known finding [5].

The entire brain development is influenced by the interaction of different genetic and nongenetic factors,

Table 1 ICC of absolute differences between related and unrelated pairs

\begin{tabular}{|c|c|c|c|c|c|c|}
\hline \multirow[t]{2}{*}{ Parameter } & \multirow[t]{2}{*}{ ICC unrelated pairs } & \multirow[t]{2}{*}{ ICC twin pairs } & \multicolumn{4}{|c|}{ ICC twins vs. unrelated pairs } \\
\hline & & & $F$ & $d f$ & $P$ & Adjusted $P$ \\
\hline A-GI right & -0.054 & 0.607 & 3.93 & 11,11 & 0.016 & 0.069 \\
\hline A-GI left & 0.139 & 0.314 & 1.42 & 11,11 & 0.29 & 0.29 \\
\hline M-GI right & 0.138 & 0.579 & 2.73 & 11,11 & 0.055 & 0.13 \\
\hline M-GI left & 0.440 & 0.646 & 1.80 & 11,11 & 0.16 & 0.29 \\
\hline \multicolumn{7}{|l|}{ Absolute volume $\mathrm{mm}^{3}$} \\
\hline Temporal lobe right & 0.201 & 0.593 & 2.56 & 11,11 & 0.065 & 0.21 \\
\hline Temporal lobe left & 0.263 & 0.559 & 2.11 & 11,11 & 0.11 & 0.24 \\
\hline Prefrontal lobe right & 0.097 & 0.766 & 6.00 & 11,11 & 0.003 & 0.032 \\
\hline Prefrontal lobe left & 0.320 & 0.740 & 3.53 & 11,11 & 0.020 & 0.092 \\
\hline Cerebellum & -0.057 & 0.768 & 8.42 & 11,11 & 0.0007 & 0.007 \\
\hline Whole brain & -0.221 & 0.821 & 10.72 & 11,11 & 0.0002 & 0.003 \\
\hline \multicolumn{7}{|l|}{ Relative volume $\%$} \\
\hline Temporal lobe right & -0.080 & 0.468 & 2.62 & 11,11 & 0.063 & 0.16 \\
\hline Temporal lobe left & -0.130 & 0.650 & 6.41 & 11,11 & 0.002 & 0.017 \\
\hline Prefrontal lobe right & -0.053 & 0.684 & 4.98 & 11,11 & 0.007 & 0.057 \\
\hline Prefrontal lobe left & 0.071 & 0.727 & 7.15 & 11,11 & 0.001 & 0.012 \\
\hline Cerebellum & -0.283 & 0.578 & 3.55 & 11,11 & 0.023 & 0.11 \\
\hline
\end{tabular}

$G I$ gyrification index, $P$ probability, $m$ mean, $s d$ standard deviation, $F F$-value, $d f$ degrees of freedom, $P P$-value, adjusted $P P$ adjusted for multiple testing according to an improved Bonferroni procedure by Simes and Hommel 
whereas formation of cortical convolutions seemed to be less genetically caused than midline structures, total brain volume, total brain surface area, and other cerebral substructures [5]. Although many authors tried to understand the genetic control of cortical regionalization, the processes underlying development of cortical convolutions remained essentially unknown $[8,19]$.

In our sample, twins showed more similarity compared to unrelated pairs. However, MZ twins did not show more similarity concerning GI and brain volumes than DZ twins. This finding may support the hypothesis of a combination of nongenetic and genetic influences on the development of cortical surface and volume. If cortical folding and development is under high genetic control, than we would assume higher similarity of gyrification and volumes in a pair of MZ twins compared to DZ twin pairs and not only in twin pairs compared to unrelated pairs.

$\mathrm{MZ}$ can be described as natural clones, developing from one oozyte which is in contrast to DZ twins. However, DZ twins are perfectly matched twin pairs, sharing parts of their genome compared to unrelated pairs. Foregoing studies have used matched, but unrelated pairs as control groups and did find more similarity in MZ twins compared to these control groups. Our results reveal a new aspect of genetic control of gyrification. One possible explanation is that DZ twins are more similar than unrelated pairs making it difficult to detect differences in GI compared to MZ twins. DZ twins develop from two different oocytes but they are siblings and have a common genetic background and a much more common prenatal environment than unrelated pairs. These conditions might countermand the previously observed higher similarity in MZ twins compared to unrelated twins. Related DZ twins seem to provide a high similarity concerning GI too and therefore a difference in lower GI similarity compared to MZ twins could not be pictured.

Cortical folding, and therefore GI, is considered to be a marker of late ontogentic brain development. In this regard, axonal connectivity and axonal dispersion are of particular importance to cortical folding, because axonal tract development begins before the onset of cortical folding and tract lesions may affect gyral patterns [20, 21]. According to the model of tension-based morphogenesis, cortical folding is influenced by differences in mechanical tensions along axons connecting different cortical regions [22]. The shape of cortical surface and therefore the gyrification pattern in humans are largely completed by birth and lead to a smooth, continuous development and the process of gyrification continues until early adulthood. In infancy maximal GI values are reached, but compensatory cortical folding maintains GI levels during continued growth [1]. Neuronal connectivity and neuroplasticity have an impact on brain functions and neuronal organization throughout adulthood and environmental factors will influence these processes.

Because GI is different from gyral pattern and gyral pattern may be more heritable than GI, we additionally compared the similarity of gyral pattern between MZ and DZ twins. Two experienced raters (A.H. and T.W.), blind to subgroup, visually inspected two corresponding prefrontal coronal slides of each twin pair and assigned this pair to the MZ or DZ twin subgroup. Although the raters differed only in two cases, only half of the slices could be assigned correct. This results means that on a first glance visual inspection of the prefrontal gyral pattern does not allow to distinguish between MZ and DZ twins, and the similarity in gyral pattern may be not higher in MZ than DZ twins. Cognitive and psychological characteristics of MZ twins seem to be more genetically determined than gyral patterns and this inconsistency might suggest that gyral pattern variations are trivial in functional terms $[3$, 23].

Regarding the conjunction with the intrauterine development, some interesting aspects of gyrification occur. MZ twins do not have the same intrauterine environment [24] and hypo- or hypergyria can be a consequence of disturbed fetal blood supply, intrauterine infection, or prenatal toxic events $[25,26]$. To evaluate GI, varying aspects of intrauterine factors and postnatal development conditions have to be respected.

From a methodical point of view, comparison of A-GI and M-GI revealed differences in the mean value of the GI (data not shown). In our analysis, the A-GI method registered higher GI value than M-GI, which is conform to other published studies. In accordance to this study, the differences between both methods could be traced back to the fact that A-GI measurement was applied to every coronal slice, whereas manual trackings were taken from three selected slices. Further methodical aspects, like higher spatial frequencies when using A-GI and the subjective judgment of the first anterior trace in the M-GI method, have to be discussed, too [13].

Our study supports the hypothesis that brain surface morphology, gyral patterns, and brain volumes underlie lesser genetic control than supposed [3,5]. It might be speculated, that the lacking difference in GI between MZ twins and DZ twins in our study could be attributed to environmental factors and other aspects of human development, whereas the observed differences between twins and unrelated pairs point to a genetic influence on brain development. This may has an important impact on psychiatric research, because changes in prefrontal gyrification and cortical folding have been found in different psychiatric conditions, like schizophrenia, OCD, and bipolar disorder $[9,10,27,28]$. 
One important limitation of our study is the relatively small number of twins with a small statistical power. Our results may not be conclusive for further interpretation and it should mentioned that the number of subjects needed to state a negative finding (comparison MZ vs. DZ twins) is substantially larger than to show a positive finding. However, we were able to present a significant difference between related and unrelated pairs, which might support our conclusions.

We are conscious that these results are preliminary and detailed information concerning genetic and environmental factors is only available to a limited extent. To summarize, we would like to suggest a hypothesis of a genetic influence on brain development with a glaring influence of environmental factors supporting a multifactor dimension of brain development.

\section{Conflict of interest None.}

Open Access This article is distributed under the terms of the Creative Commons Attribution Noncommercial License which permits any noncommercial use, distribution, and reproduction in any medium, provided the original author(s) and source are credited.

\section{References}

1. Armstrong E, Schleicher A, Omran H, Curtis M, Zilles K (1995) The ontogeny of human gyrification. Cereb Cortex 5:56-63

2. Magnotta VA, Andreasen NC, Schultz SK, Harris G, Cizadlo T, Heckel D, Nopoulos P, Flaum M (1999) Quantitative in vivo measurement of gyrification in the human brain: changes associated with aging. Cereb Cortex 9:151-160

3. Bartley AJ, Jones DW, Weinberger DR (1997) Genetic variability of human brain size and cortical gyral patterns. Brain $120(\mathrm{Pt}$ 2):257-269

4. Lohmann G, von Cramon DY, Steinmetz H (1999) Sulcal variability of twins. Cereb Cortex 9:754-763

5. Mohr A, Weisbrod M, Schellinger P, Knauth M (2004) The similarity of brain morphology in healthy monozygotic twins. Brain Res Cogn Brain Res 20:106-110

6. Biondi A, Nogueira H, Dormont D, Duyme M, Hasboun D, Zouaoui A, Chantome M, Marsault C (1998) Are the brains of monozygotic twins similar? A three-dimensional MR study. AJNR Am J Neuroradiol 19:1361-1367

7. White T, Andreasen NC, Nopoulos P (2002) Brain volumes and surface morphology in monozygotic twins. Cereb Cortex $12: 486-493$

8. Toro R, Burnod Y (2005) A morphogenetic model for the development of cortical convolutions. Cereb Cortex 15:1900-1913

9. Falkai P, Honer WG, Kamer T, Dustert S, Vogeley K, SchneiderAxmann T, Dani I, Wagner M, Rietschel M, Muller DJ, Schulze TG, Gaebel W, Cordes J, Schonell H, Schild HH, Block W, Traber F, Steinmetz H, Maier W, Tepest R (2007) Disturbed frontal gyrification within families affected with schizophrenia. J Psychiatr Res 41:805-813

10. Wobrock T, Gruber O, McIntosh AM, Kraft S, Klinghardt A, Scherk H, Reith W, Schneider-Axmann T, Lawrie SM, Falkai P, Moorhead TW (2010) Reduced prefrontal gyrification in obsessive-compulsive disorder. Eur Arch Psychiatry Clin Neurosci 260(6):455-464

11. Zilles K, Armstrong E, Schleicher A, Kretschmann HJ (1988) The human pattern of gyrification in the cerebral cortex. Anat Embryol 179:173-179

12. Harris JM, Yates S, Miller P, Best JJ, Johnstone EC, Lawrie SM (2004) Gyrification in first-episode schizophrenia: a morphometric study. Biol Psychiatry 55:141-147

13. Moorhead TW, Harris JM, Stanfield AC, Job DE, Best JJ, Johnstone EC, Lawrie SM (2006) Automated computation of the gyrification index in prefrontal lobes: methods and comparison with manual implementation. Neuroimage 31:1560-1566

14. Vogeley K, Schneider-Axmann T, Pfeiffer U, Tepest R, Bayer TA, Bogerts B, Honer WG, Falkai P (2000) Disturbed gyrification of the prefrontal region in male schizophrenic patients: a morphometric postmortem study. Am J Psychiatry 157:34-39

15. Ashburner J, Friston KJ (2000) Voxel-based morphometry-the methods. Neuroimage 11:805-821

16. Bonnici HM, William T, Moorhead J, Stanfield AC, Harris JM, Owens DG, Johnstone EC, Lawrie SM (2007) Pre-frontal lobe gyrification index in schizophrenia, mental retardation and comorbid groups: an automated study. Neuroimage 35:648-654

17. Vogeley K, Tepest R, Pfeiffer U, Schneider-Axmann T, Maier W, Honer WG, Falkai P (2001) Right frontal hypergyria differentiation in affected and unaffected siblings from families multiply affected with schizophrenia: a morphometric MRI study. Am J Psychiatry 158:494-496

18. Hommel G (1988) A comparison of two modified Bonferroni procedures. Biometrika 75:383-386

19. Pallas SL (2001) Intrinsic and extrinsic factors that shape neocortical specification. Trends Neurosci 24:417-423

20. Rakic P (2000) Setting the stage of cognition: genesis of the primate cerebral cortex. MIT Press, London

21. Rakic P (1988) Specification of cerebral cortical areas. Science 241:170-176

22. Van Essen DC (1997) A tension-based theory of morphogenesis and compact wiring in the central nervous system. Nature 385:313-318

23. Plomin R, Owen MJ, McGuffin P (1994) The genetic basis of complex human behaviors. Science 264:1733-1739

24. Hrubec Z, Robinette CD (1984) The study of human twins in medical research. N Engl J Med 310:435-441

25. Goldman-Rakic PS (1980) Morphological consequences of prenatal injury to the primate brain. Prog Brain Res 53:1-19

26. Jellinger K, Rett A (1976) Agyria-pachygyria (lissencephaly syndrome). Neuropadiatrie 7:66-91

27. McIntosh AM, Moorhead TW, McKirdy J, Hall J, Sussmann JE, Stanfield AC, Harris JM, Johnstone EC, Lawrie SM (2009) Prefrontal gyral folding and its cognitive correlates in bipolar disorder and schizophrenia. Acta Psychiatr Scand 119:192-198

28. Schultz CC, Koch K, Wagner G, Roebel M, Schachtzabel C, Nenadic I, Albrecht C, Reichenbach JR, Sauer H, Schlosser RG (2010) Psychopathological correlates of the entorhinal cortical shape in schizophrenia. Eur Arch Psychiatry Clin Neurosci 260:351-358 